TITLE:

\title{
Production of rare sugars from common sugars in subcritical aqueous ethanol.
}

$\operatorname{AUTHOR}(\mathrm{S}):$

Gao, Da-Ming; Kobayashi, Takashi; Adachi, Shuji

CITATION:

Gao, Da-Ming ...[et al]. Production of rare sugars from common sugars in subcritical aqueous ethanol.. Food chemistry 2015, 175: 465-470

ISSUE DATE:

2015-05-15

URL:

http://hdl.handle.net/2433/193287

RIGHT:

(C) 2014 Elsevier Ltd.; This is not the published version. Please cite only the published version.; この論文は出版社版でありません。引用の際に は出版社版をご確認ご利用ください。 


\title{
Production of rare sugars from common sugars in subcritical aqueous ethanol
}

\author{
Da-Ming Gao, Takashi Kobayashi and Shuji Adachi*
}

Division of Food Science and Biotechnology, Graduate School of Agriculture, Kyoto University, Sakyo-ku, Kyoto 606-8502, Japan

\begin{abstract}
A new isomerization reaction was developed to synthesize rare ketoses. D-Tagatose, D-xylulose, and D-ribulose were obtained in the maximum yields of $24 \%, 38 \%$, and $40 \%$, respectively, from the corresponding aldoses, D-galactose, D-xylose, and D-ribose, by treating the aldoses with $80 \%(\mathrm{v} / \mathrm{v})$ subcritical aqueous ethanol at $180{ }^{\circ} \mathrm{C}$. The maximum productivity of $\mathrm{D}$-tagatose was ca. 80 $\mathrm{g} /(\mathrm{L} \cdot \mathrm{h})$. Increasing the concentration of ethanol significantly increased the isomerization of D-galactose. Variation in the reaction temperature did not significantly affect the production of D-tagatose from D-galactose. Subcritical aqueous ethanol converted both 2,3-threo and 2,3-erythro aldoses to the corresponding C-2 ketoses in high yields. Thus, the treatment of common aldoses in subcritical aqueous ethanol can be regarded as a new method to synthesize the corresponding rare sugars.
\end{abstract}

Keywords: D-tagatose; D-xylulose; D-ribulose; ketose-aldose isomerization; subcritical aqueous ethanol

\section{Introduction}

Rare sugars have attracted much attention in food, chemical, and fuel industries. There are many rare ketoses such as D-tagatose, D-xylulose, and D-ribulose. Because D-tagatose has low energy (almost $0 \mathrm{~kJ} / \mathrm{g}$ of food energy) and can be used as a drug to control type- 2 diabetes and obesity, it can be used as a sweetener substitute for sucrose (Levin, Zehner, Saunders, \& Beadle, 1995; Lu, Levin, \& Donner, 2008). Besides, dietary restriction of energy by the intake of $\mathrm{D}$-tagatose reduces the incidence of neoplastic lesions and significantly extends the maximum life span (Levin, Zehner, Saunders, \& Beadle, 1995). $\mathrm{D}$-Xylulose is not only an energy resource for growth but also can be used in the pharmaceutical chemistry (Beerens, Desmet, \& Soetaert, 2012) and to synthesize furfural as a safe flavor ingredient in food (Adams et al., 1997). There are few reports on the physiological effects of D-ribulose because of the difficulty in its production and its high cost. D-Ribulose is an important material to synthesize nucleosides (Marugg, Tromp, KuylYeheshiely, van der Marel, \& van Boom, 1986). However, among the possible isomers, only seven pentoses and hexoses (D-glucose, D-mannose, D-fructose, D-xylose, D-galactose, D-ribose, and L-arabinose) are found in sufficient quantities in nature to satisfy commercial needs (Gunther et al., 2012). The conversion of these abundant common sugars to rare sugars has become necessary and attracted much attention.

The isomerization of common sugars to rare sugars has been mainly performed by alkali, metal, and enzymatic catalyses. In alkali-catalyzed isomerization reactions, glucose-type monosaccharides (2,3-threo-type) could be easily isomerized to the corresponding C-2 ketoses such as glucose to fructose isomerization; however, mannose-type monosaccharides (2,3erythro-type) were isomerized to the C-2 ketoses in low yields, such as mannose to fructose isomerization (Khadem, Ennifar, \& Isbell, 1989). Using alkali-catalyzed isomerization, D-tagatose, $\mathrm{D}$-xylulose, and D-ribulose could be obtained in low yields from the corresponding glucose-type aldoses (Khadem, Ennifar, \& Isbell, 1989; Mendicino, 1960; Tipson \& Brady Jr, 1969). However, alkali-catalyzed isomerization usually results in many by-products due to various side reactions, and this process often requires tedious purification steps.

The product distribution of metal-catalyzed isomerization reactions has a complicated dependence on the type of metal ions, substrate saccharides, cosolvent, and support of metal ions. By using $N, N, N^{\prime}, N^{\prime}$-tetramethylenediamine and calcium chloride together in a methanolic solution, $\mathrm{Ca}^{2+}$ converted $\mathrm{D}$-galactose to D-tagatose in $48 \%$ yield (Yamauchi, Fukushima, Yanagihara, Osanai, \& Yoshikawa, 1990). However, D-galactose concentration was too low for practical applications. Recently, D-xylulose was synthesized in ca. $30 \%$ yield from D-xylose with an initial concentration of $10 \mathrm{wt} \%$ using a tin-containing zeolite as the catalyst (Choudhary, Caratzoulas, \& Vlachos, 2013). The preparation of tin-containing zeolite takes many days, and the reaction mixture may contain heavy metals. The synthesis of
D-ribulose using metal catalysts has not been satisfactory because of the low yield or troublesome postprocessing. An expedient synthesis of $D$-ribulose was performed by treating D-arabinose with a $\mathrm{Pd} / \mathrm{C}$ catalyst, leading to an overall yield of $20 \%$ (Vuorinen \& Serianni, 1990). However, D-arabinose is an expensive rare sugar.

Biotransformations of abundant saccharides to rare saccharides have been performed primarily using aldose-ketose isomerases, epimerases, and polyol dehydrogenases (Izumori, 2006). L-Arabinose isomerase (EC 5.3.1.4) has been commonly used to directly synthesize D-tagatose from D-galactose on a large scale (Lim, Kim, \& Oh, 2007; Jørgensen, Hansen, \& Stougaard, 2004). By utilizing an L-arabinose isomerase, 370 $\mathrm{g} / \mathrm{L}$ D-tagatose was obtained from $500 \mathrm{~g} / \mathrm{L}$ D-galactose with a reaction time of $24 \mathrm{~h}(\mathrm{Lim}, \mathrm{Kim}, \& \mathrm{Oh}, 2007)$. Xylose isomerase (EC 5.3.1.5), which is generally used in the production of high-fructose corn syrup from glucose, can also synthesize D-xylulose directly from D-xylose. However, the chemical equilibrium is not favorable for D-xylulose in the enzymatic isomerization because D-xylulose was obtained only in ca. $25 \%$ overall yield at room temperature (Chiang, Hsiao, Ueng, \& Tsao, 1981). Attempts to prepare D-ribulose to allow commercial production have not been successful even by utilizing enzymes because of the low yield and complicated pretreatment and post-purification.

Therefore, it is necessary to develop a new method to synthesize these rare sugars simply and efficiently, preferably by one-step isomerization. It was reported that the isomerization of monosaccharides occurred in subcritical water, in which aldoses easily isomerized to the corresponding C-2 ketoses (Lü \& Saka, 2012; Usuki, Kimura, \& Adachi, 2007). The kinetic analysis showed that among the isomerization reactions of glucose, mannose, and fructose, mannose most easily isomerized to fructose (Usuki, Kimura, \& Adachi, 2007). However, the yields of the derived monosaccharides were still low despite varying the reaction temperature (Usuki, Kimura, \& Adachi, 2007). However, the reaction equilibrium constants of the monosaccharide isomerization of aldoses to ketoses became larger with increasing reaction temperature (Moliner, Roman-Leshkov, \& Davis, 2010). Adding an organic solvent often changes the apparent chemical equilibrium and reaction rate. Among the organic solvents investigated, ethanol can be safely used in food manufacturing processes. Vuorinen and Sjostrom (1982) reported that when the isomerization of glucose and fructose was carried out in aqueous ethanol in the presence of sodium hydroxide, the rate constants of glucose to fructose isomerization, and vice versa, significantly increased with increasing ethanol concentration, and the yield of fructose in 70 $\mathrm{wt} \%$ ethanol almost tripled compared to that obtained in water (Vuorinen \& Sjostrom, 1982). Similar effects of ethanol were also observed in enzymatic isomerization, and the equilibrium yield of fructose from glucose became ca. $60 \%$ in $90 \mathrm{wt} \%$ aqueous ethanol (Visuri \& Klibanov, 1987). We also reported 
that the addition of ethanol promoted the isomerization of glucose to fructose during the hydrolysis of sucrose in subcritical aqueous ethanol (Gao, Kobayashi, \& Adachi, 2014).

In this context, we investigated the isomerization of two glucose-type aldoses, D-galactose and D-xylose, and a mannose-type aldose, D-ribose, in subcritical aqueous ethanol for efficiently producing the corresponding rare C-2 ketoses, D-tagatose, D-xylulose, and D-ribulose.

\section{A2. Materials and methods}

\subsection{Materials}

D-Xylose, D-lyxose, D-galactose, D-tagatose, D-talose, and D-arabinose were purchased from Wako Pure Chemical Industries (Osaka, Japan). D-Xylulose and D-ribose were purchased from Sigma-Aldrich (Tokyo, Japan). Because the isomerization of only the D-enantiomers was focused in this study, the prefix, D-, of all the saccharides was omitted hereafter.

\subsection{Isomerization of common sugars to rare sugars in subcritical} aqueous ethanol

The isomerization reactions were carried out in a coiled stainless steel tubular reactor $(0.8 \mathrm{~mm}$ I.D. $\times 1.0 \mathrm{~m}$ length $)$ heated in a silicone oil bath. To terminate the reaction, the reactor effluent was directly introduced to a stainless steel tube immersed in an ice bath. The residence time was set in the range $30-500 \mathrm{~s}$. The reaction temperature was set at $180{ }^{\circ} \mathrm{C}$ for the treatment of xylose and ribose and at $160-200{ }^{\circ} \mathrm{C}$ for the treatment of galactose. Xylose and ribose were treated in $80 \%$ (v/v) aqueous ethanol, and the ethanol concentration was changed from 0 to $80 \%(\mathrm{v} / \mathrm{v})$ for the treatment of galactose. Each monosaccharide was dissolved in distilled water, and the resulting solution was mixed with ethanol to obtain a solution at the predetermined concentration. Both the concentrations of xylose and ribose were adjusted at $0.5 \%(\mathrm{w} / \mathrm{v})$, and the galactose concentration was $0.5-8.5 \%$. The pressure inside the reactor was regulated at $10 \mathrm{MPa}$ using a back-pressure valve (Upchurch Scientific Inc., Oak Harbor, WA, USA).

The residence time was calculated according to the method reported in our previous study (Gao, Kobayashi, \& Adachi, 2014).

\subsection{HPLC analysis}

The reactor effluent was collected into a test tube, and the liquid samples were analyzed by high-performance liquid chromatography (HPLC). The HPLC system was equipped with an LC-10ADVP pump (Shimadzu, Kyoto, Japan), a COSMOSIL Sugar-D column (4.6 mm I.D. $\times 250 \mathrm{~mm}$ length, Nacalai Tesque, Kyoto, Japan), and a refractometer (RI-101, Showa Denko, Tokyo, Japan). A mixture of water and acetonitrile (20:80, v/v) was used as the eluent at a flow rate of $1 \mathrm{~mL} / \mathrm{min}$. The column temperature was regulated at $30^{\circ} \mathrm{C}$ using a CTO-10A VP column oven (Shimadzu). Lyxose was analyzed by a combination of two columns, a COSMOSIL Sugar-D column and a $\mathrm{Ca}^{2+}$ ion-exchange column, SUPELCOGEL CA (7.8 mm I.D. $\times 300$ $\mathrm{mm}$ length, Sigma-Aldrich, Tokyo, Japan), under the same conditions as for the other saccharides.

\subsection{Purification and NMR confirmation of rare sugars}

The reactor effluent (ca. $200 \mathrm{~mL}$ ) was concentrated to ca. 1 $\mathrm{mL}$ using a rotary evaporator (OSR-B, Techno Sigma, Okayama, Japan). The concentrated solution was treated using a Strata C18-E cartridge column (55 $\mu \mathrm{m}, 70 \AA$, Shimadzu) as pretreatment. A solution of $50 \%(\mathrm{v} / \mathrm{v})$ aqueous methanol was used as the eluent. The solvent in the effluent from the cartridge was evaporated using a rotary evaporator. The purification of the target saccharide was performed using a COSMOSIL Sugar-D column $(10 \mathrm{~mm}$ I.D. $\times 250 \mathrm{~mm}$ length, Nacalai Tesque $)$ using $95 \%(\mathrm{v} / \mathrm{v})$ acetonitrile as the eluent at a flow rate of $3 \mathrm{~mL} / \mathrm{min}$.

The ${ }^{1} \mathrm{H}$ NMR analyses of the purified saccharides were carried out using Ascend $400 \mathrm{MHz}$ NMR spectrometer (Bruker Japan, Osaka, Japan) with $\mathrm{D}_{2} \mathrm{O}$ as the solvent. Acetonitrile $\left(\delta_{\mathrm{H}}=2.06\right.$ $\mathrm{ppm}$ ) was used as the internal standard. The ${ }^{1} \mathrm{H}$ NMR spectra of the purified tagatose, xylulose, and ribulose were compared with those of the commercial samples or with literature datum (Vuorinen \& Serianni, 1990). Confirmation of arabinose, lyxose, and talose was performed by comparing their retention times in the HPLC chromatograms with the commercial ones due to the low yields to obtain the purified products for the NMR analysis.

\subsection{Solubility of galactose in aqueous ethanol}

The solubility of galactose in aqueous ethanol of different concentrations was measured at $25{ }^{\circ} \mathrm{C}$. A sufficient amount of galactose was added to aqueous ethanol, and the mixture was stirred at $25{ }^{\circ} \mathrm{C}$ using a digital stirring plate (Koike Precision Instruments, Kyoto, Japan) to reach the equilibrium. The concentration of galactose in the supernatant was then measured using the abovementioned HPLC system.

\section{Results and discussion}

3.1 Production of tagatose, xylulose, and ribulose from the corresponding common sugars

Isomerization of the common sugars was performed in subcritical aqueous ethanol. Figure 1 shows the typical HPLC chromatograms of the reaction mixtures containing rare sugars formed from the corresponding common sugars. By the treatment of common sugars in subcritical aqueous ethanol, several peaks, which would correspond to the respective rare sugars, were observed. By comparing the NMR spectra, tagatose and xylulose were formed during the treatment (Suppl. figs. 1 and 2). Formation of ribulose was confirmed by comparing the NMR spectrum with that in the literature by Vuorinen \& Serianni (1990) as shown in Suppl. fig. 3.

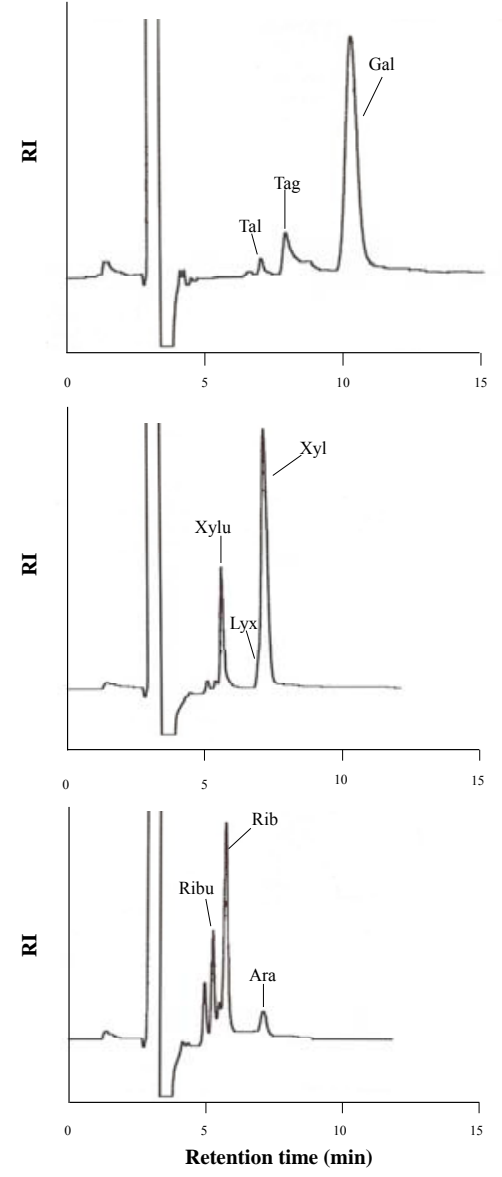

Fig. 1. HPLC chromatograms of the reaction mixtures containing rare sugars formed from (A) galactose, (B) xylose, and (C) ribose in $80 \%$ $(\mathrm{v} / \mathrm{v})$ aqueous ethanol at the residence time of $300 \mathrm{~s}$. The feed concentration of each substrate was $0.5 \%(\mathrm{w} / \mathrm{v})$. 
Synthesis of rare sugars

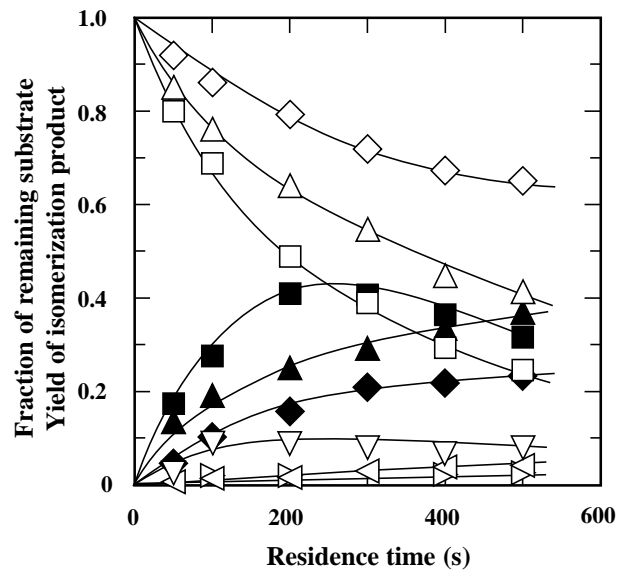

Fig. 2. Isomerization processes of $(\diamond)$ galactose to $(\diamond)$ tagatose and $(\triangleleft)$ talose, $(\triangle)$ xylose to $(\boldsymbol{\Delta})$ xylulose and $(\triangleright)$ lyxose, and $(\square)$ ribose to $(\boldsymbol{\square})$ ribulose and $(\nabla)$ arabinose in $80 \%(\mathrm{v} / \mathrm{v})$ subcritical aqueous ethanol at $180{ }^{\circ} \mathrm{C}$. The concentration of each substrate was $0.5 \%(\mathrm{w} / \mathrm{v})$. Yield was defined as the ratio of the concentration of the obtained saccharide to that of the initial concentration of the substrate.

It was reported that mannose and glucose can isomerize each other in subcritical aqueous ethanol (Gao, Kobayashi, \& Adachi, in press), and that arabinose, lyxose, and talose can be also formed in subcritical water from ribose, xylose, and galactose, respectively (Lü \& Saka, 2012). In addition, the retention times of these rare sugars in the HPLC chromatograms corresponded with those of the commercial ones. From these results, arabinose, lyxose, and talose would be formed in subcritical aqueous ethanol.

Figure 2 shows the time courses of the isomerization of galactose to tagatose and talose, xylose to xylulose and lyxose, and ribose to ribulose and arabinose in $80 \%(\mathrm{v} / \mathrm{v})$ aqueous ethanol at $180{ }^{\circ} \mathrm{C}$. The conversions of galactose, xylose, and ribose increased with increasing residence time and reached ca. $32 \%, 60 \%$, and $75 \%$, respectively, at a residence time of $500 \mathrm{~s}$. The yield of tagatose increased with the consumption of galactose and reached ca. $24 \%$ at a residence time of $500 \mathrm{~s}$. In contrast, the selectivity of tagatose first remained high $(>80 \%)$ until the residence time of $200 \mathrm{~s}$ and then decreased at longer residence times, where the selectivity was defined as the molar ratio of the obtained product to the consumed substrate. Overall, the selectivity of tagatose was $>70 \%$ within the treatment time. Talose, the C-2 epimer of galactose, was also synthesized from galactose; however, its yield was $<5 \%$.

Xylulose and lyxose were synthesized from xylose, and their yields at the residence time of $500 \mathrm{~s}$ were ca. $38 \%$ and $2 \%$, respectively. The selectivity of xylulose gradually decreased with the consumption of xylose; however, it was $>60 \%$ at the investigated residence times.

Ribose decreased most rapidly among the three substrates. Some peaks were observed in the HPLC chromatogram of the reaction mixture (Fig. 1), and arabinose was obtained in $<10 \%$ yield at the investigated residence times. On the other hand, ribulose was obtained in high yields. A maximum yield of ca. $40 \%$ was first obtained at a residence time of $200 \mathrm{~s}$; however, the yield then decreased at longer residence times. At the residence time of $500 \mathrm{~s}$, only $30 \%$ of the ribulose remained, indicating that the obtained ribulose was further decomposed to other compounds and that prolonging the reaction time promoted the decomposition reaction. Therefore, the optimal residence time to synthesize ribulose was set at ca. $200 \mathrm{~s}$.

The isomerization behaviors of these three aldoses in subcritical aqueous ethanol were unique in the following four aspects compared to the alkali-catalyzed, metal-catalyzed, and enzymatic isomerization reactions (Khadem, Ennifar, \& Isbell, 1989; Yamauchi, Fukushima, Yanagihara, Osanai, \& Yoshikawa, 1990; Jørgensen, Hansen, \& Stougaard, 2004). 1) Subcritical aqueous ethanol converted both glucose-type (2,3-threo) and mannose-type (2,3-erythro) aldoses to the corresponding $\mathrm{C}-2$ ketoses in high yields and selectivity. This indicates that subcritical aqueous ethanol showed different substrate-dependent chemoselective aldose-ketose isomerizations. In particular, ribulose could be synthesized in high yields from ribose rather than from arabinose. 2) The isomerization reactions between aldoses and ketoses were difficult. 3) The apparent reaction equilibrium shifted towards the formation of ketoses at high temperatures. 4) The production process is one-step and only requires a very simple reactor and safety solvent. In addition, recovery of catalysts was not required during the post-treatment, and ethanol can be easily recovered by evaporation for its reuse. However, there are some disadvantages. Because ethanol is contained in the reaction mixture, saturated concentration of sugars is lower than in water, indicating that the product concentration would not be high.

\subsection{Temperature dependence of galactose isomerization}

Figure 3 shows the effect of reaction temperature on the isomerization of galactose in $80 \%(\mathrm{v} / \mathrm{v})$ aqueous ethanol. The conversion of galactose increased with increasing reaction temperature. When the temperature exceeded $180{ }^{\circ} \mathrm{C}$, galactose underwent rapid conversion with a low yield of tagatose. The increase in the reaction temperature from 160 to $180{ }^{\circ} \mathrm{C}$ increased galactose conversion only by ca. $13 \%$ at a residence time of $500 \mathrm{~s}$. However, when the reaction temperature was increased from 180 to $190{ }^{\circ} \mathrm{C}$, the conversion of galactose also increased from ca. $34 \%$ to $71 \%$. On the other hand, the initial reaction rates for the formation of tagatose and talose increased with increasing reaction temperature.

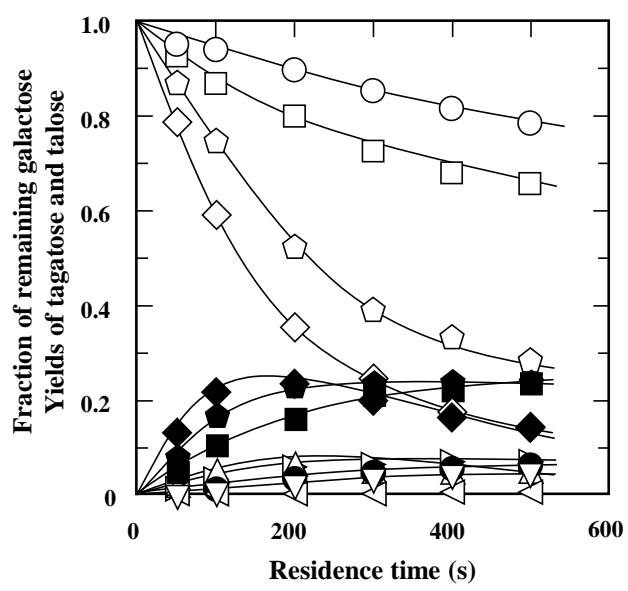


$(\diamond) 200{ }^{\circ} \mathrm{C}$, on the isomerization behavior of galactose in $80 \%(\mathrm{v} / \mathrm{v})$ aqueous ethanol with a galactose feed concentration of $0.5 \%(\mathrm{w} / \mathrm{v})$. Symbols $\bullet, \mathbf{a}, \boldsymbol{D}$, and $\diamond$ represent tagatose and symbols $\triangleleft, \nabla, \nabla$, and $\triangle$ represent talose synthesized at $160,180,190$, and $200{ }^{\circ} \mathrm{C}$, respectively.

The yield of tagatose did not exceed $8 \%$ at $160{ }^{\circ} \mathrm{C}$, but increased to ca. $24 \%$ at $180{ }^{\circ} \mathrm{C}$ at a residence time of $500 \mathrm{~s}$. However, increasing the temperature to 190 and $200{ }^{\circ} \mathrm{C}$ increased the yield of tagatose at a residence time shorter than $300 \mathrm{~s}$, albeit with lower selectivity. The selectivity of tagatose obtained at $200{ }^{\circ} \mathrm{C}$ for $200 \mathrm{~s}$ was approximately half of that obtained at $180{ }^{\circ} \mathrm{C}$ at a residence time of $500 \mathrm{~s}$. The results indicate that increasing reaction temperature beyond $180{ }^{\circ} \mathrm{C}$ favored the decomposition reaction and caused a large loss of the total saccharide yield. The maximum yields of tagatose at 180 , 190 , and $200{ }^{\circ} \mathrm{C}$ were almost the same; thus, a reaction temperature higher than $180{ }^{\circ} \mathrm{C}$ did not increase the yield of tagatose. 
Synthesis of rare sugars

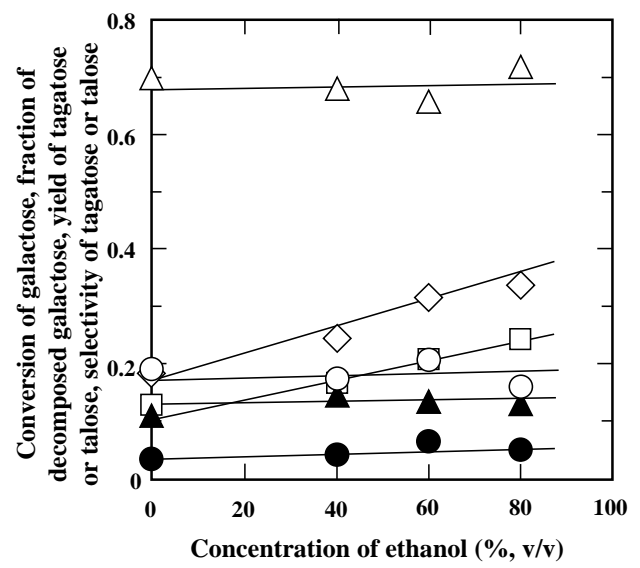

Fig. 4. Effect of ethanol concentration on $(\diamond)$ the conversion of galactose, $(\square)$ yield of tagatose, $(\triangle)$ selectivity of tagatose, $(\bullet)$ yield of talose, $(\bigcirc)$ selectivity of talose, and $(\mathbf{\Lambda})$ fraction of decomposed galactose. Galactose with a feed concentration of $0.5 \%(\mathrm{w} / \mathrm{v})$ was treated at $180{ }^{\circ} \mathrm{C}$ and at a residence time of $500 \mathrm{~s}$. The fraction of decomposed galactose was defined as the ratio of the concentration of the side products to that of the consumed galactose.

\subsection{Effect of ethanol concentration on the isomerization of galactose}

The isomerization of galactose significantly increased with increasing ethanol concentration (Fig. 4). When the reaction medium was changed from subcritical water to $80 \%(\mathrm{v} / \mathrm{v})$ subcritical aqueous ethanol for the isomerization of galactose at $180{ }^{\circ} \mathrm{C}$ for $500 \mathrm{~s}$, the conversion of galactose and the yield of tagatose increased to ca. $34 \%$ and $24 \%$, respectively. However, the change in ethanol concentration barely affected the selectivity of tagatose and the fraction of decomposed galactose. This result indicates that increasing the concentration of ethanol promoted both the isomerization and decomposition of galactose. In contrast, the yield and selectivity of talose were hardly affected by the change in ethanol concentration. Vourinen and Sjostrom (1982) also reported that ethanol promoted both the isomerization and decomposition of monosaccharides in an aqueous alkaline solution, and they attributed the promotion ability of ethanol to increasing in ionization constant of monosaccharides. In this study, there is a possibility that the similar mechanism acted on the promotion of the isomerization. Ethanol also promotes the isomerization of saccharides by changing the conformation and configuration of the monosaccharides, thus shifting the apparent reaction equilibrium to product formation (Angyal, 1969). The contribution of ethanol to the isomerization of these saccharides in subcritical aqueous ethanol is still unclear. Under subcritical conditions, the role of ethanol in promoting the isomerization reaction may be more complicated.



Fig. 5. Solubility of galactose in aqueous ethanol at $25^{\circ} \mathrm{C}$.
Table 1 Effect of galactose feed concentration on its isomerization.

\begin{tabular}{ccccccc}
\hline $\begin{array}{c}\text { Feed concentration } \\
\text { of galactose } \\
(\%, \mathrm{w} / \mathrm{v})\end{array}$ & $\begin{array}{c}\text { Galactose } \\
\text { conversion } \\
(\%)\end{array}$ & $\begin{array}{c}\text { Yield of } \\
\text { talose } \\
(\%)\end{array}$ & $\begin{array}{c}\text { Yield of } \\
\text { tagatose } \\
(\%)\end{array}$ & $\begin{array}{c}\text { Selectivity } \\
\text { of tagatose } \\
(\%)\end{array}$ & $\begin{array}{c}\text { Productivity of } \\
\text { tagatose } \\
(\mathrm{g} / \mathrm{L} \cdot \mathrm{h})\end{array}$ & $\begin{array}{c}\text { Total yield } \\
\text { of sugars } \\
(\%)\end{array}$ \\
\hline 0.5 & 32 & 7 & 21 & 66 & 8 & 96 \\
1.0 & 29 & 5 & 20 & 69 & 14 & 96 \\
2.0 & 29 & 4 & 20 & 69 & 29 & 95 \\
5.0 & 24 & 3 & 14 & 58 & 50 & 93 \\
8.5 & 23 & 3 & 13 & 57 & 80 & 93 \\
\hline
\end{tabular}

The isomerization reaction was carried out in $60 \%(\mathrm{v} / \mathrm{v})$ subcritical aqueous ethanol at $180{ }^{\circ} \mathrm{C}$ and at the residence time of $500 \mathrm{~s}$. The total yield of sugars was defined as the sum of the remaining fraction of galactose and yields of tagatose and talose.

\subsection{Isomerization of galactose at different feed concentrations}

It is desirable to feed galactose in high concentrations without decreasing the yield and selectivity of tagatose during the isomerization. The solubility of galactose in aqueous ethanol was measured at $25{ }^{\circ} \mathrm{C}$ (Fig. 5). The addition of ethanol significantly decreased the solubility of galactose from ca. $36 \%(\mathrm{w} / \mathrm{v})$ to $1.0 \%$ by changing the solvent from water to $80 \%(\mathrm{v} / \mathrm{v})$ aqueous ethanol. Considering the yield of tagatose and the solubility of galactose, the reaction was carried out in $60 \%(\mathrm{v} / \mathrm{v})$ ethanol at $180{ }^{\circ} \mathrm{C}$ to investigate the effect of galactose feed concentration on its isomerization. The solubility of galactose was ca. 9.3\% $(\mathrm{w} / \mathrm{v})$ in $60 \%(\mathrm{v} / \mathrm{v})$ aqueous ethanol at $25{ }^{\circ} \mathrm{C}$. To avoid the precipitation of galactose, the maximum feed concentration was selected as $8.5 \%(\mathrm{w} / \mathrm{v})$. Other feed concentrations were $0.5 \%$ $(\mathrm{w} / \mathrm{v}), 1.0 \%, 2.0 \%$, and $5.0 \%$.

The conversion of galactose decreased at its concentrations of $5.0 \%$ and $8.5 \%(\mathrm{w} / \mathrm{v})$ (Table 1). For the treatment at $180{ }^{\circ} \mathrm{C}$ for $500 \mathrm{~s}$, the yield and selectivity of tagatose decreased to ca. $13 \%$ and $57 \%$, respectively, and the conversion of galactose decreased to ca. $23 \%$ by changing the galactose feed concentration from $0.5 \%(\mathrm{w} / \mathrm{v})$ to $8.5 \%$. However, the maximum productivity of tagatose, ca. $80 \mathrm{~g} /(\mathrm{L} \cdot \mathrm{h})$, was realized at a feed concentration of $8.5 \%(\mathrm{w} / \mathrm{v})$. In the enzymatic isomerization, the highest productivity of tagatose was reported to be $15.3 \mathrm{~g} /(\mathrm{L} \cdot \mathrm{h})$ using an L-arabinose isomerase ( $\mathrm{Lim}, \mathrm{Kim}, \& \mathrm{Oh}, 2007)$. The results indicate that the subcritical aqueous ethanol treatment is promising for the practical synthesis of tagatose. The reasons for the decrease in the galactose conversion at high feed concentrations are presently unclear in subcritical aqueous ethanol at high temperature.

In conclusion, three rare ketoses, tagatose, xylulose, and ribulose, were preferably synthesized by one-step isomerization from the corresponding aldoses using subcritical aqueous ethanol. In contrast, the rare aldoses, talose, lyxose, and arabinose, were synthesized in low yields. These facts clearly demonstrate that ketoses can be selectively synthesized from the corresponding aldoses using subcritical aqueous ethanol.

\section{Acknowledgement}

This study was partly supported by the Tojuro Iijima Foundation for Food Science and Technology (No. H26-18; D.G.) and by JSPS KAKENHI (No. 26870296; T. K.).

\section{References}

Adams, T. B., Doull, J., Goodman, J. I., Munro, I. C., Newberne, P., Portoghese, P. S., et al. (1997). The FEMA GRAS assessment of furfural used as a flavour ingredient. Food and Chemical Toxicology, 35(8), 739-751.

Angyal, S. J. (1969). The composition and conformation of sugars in solution. Angewandte Chemie International Edition in English, 8(3), 157-166.

Beerens, K., Desmet, T., \& Eoetaert, W. (2012). Enzymes for the biocatalytic production of rare sugars. Journal of Industrial Microbiology and Biotechnology, 39(6), 823-834.

Chiang, L. C., Hsiao, H. Y., Ueng, P. P., \& Tsao, G. T. (1981). Enzymatic and microbial preparation of D-xylulose from 
Synthesis of rare sugars

D-xylose. Applied and Environmental Microbiology, 42(1), 66-69.

Choudhary, V., Caratzoulas, S., \& Vlachos, D. G. (2013). Insights into the isomerization of xylose to xylulose and lyxose by a Lewis acid catalyst. Carbohydrate Research, 368, 89-95.

Gao, D., Kobayashi, T., \& Adachi, S. (2014). Kinetics of sucrose hydrolysis in a subcritical water-ethanol mixture. Journal of Applied Glycoscience, 61, 9-13.

Gao, D., Kobayashi, T., \& Adachi, S. Promotion or suppression of glucose isomerization in subcritical aqueous straight- and branched-chain alcohols. Bioscience, Biotechnology, and Biochemistry, in press, Doi: 10.1080/09168451.2014.973366.

Gunther, W. R., Wang, Y. R., Ji, Y. W., Michaelis, V. K., Hunt, S. T., \& Griffin, R. G. (2012). Sn-Beta zeolites with borate salts catalyse the epimerization of carbohydrates via an intramolecular carbon shift. Nature Communications, 3, 1-8.

Izumori, K. (2006). Izumoring: a strategy for bioproduction of all hexoses. Journal of Biotechnology, 124 (4), 717-722.

Jørgensen, F., Hansen, O. C., \& Stougaard, P. (2004). Enzymatic conversion of D-galactose to D-tagatose: heterologous expression and characterisation of a thermostable L-arabinose isomerase from Thermoanaerobacter mathranii. Applied Microbiology and Biotechnology, 64, 816-822.

Khadem, H. S. E., Ennifar, S., \& Isbell, H. S. (1989). Evidence of stable hydrogen-bonded ions during isomerization of hexoses in alkali. Carbohydrate Research, 185(1), 51-59.

Levin, G. V., Zehner, L. R., Saunders, J. P., \& Beadle, J. R. (1995). Sugar substitutes: their energy values, bulk characteristics, and potential health benefits. American Journal of Clinical Nutrition, 62(5), 1161s-1168s.

Lim, B. C., Kim, H. J., \& Oh, D. K. (2007). A high production of D-tagatose by the addition of boric acid. Biotechnology Progress, 23(4), 824-828.

Lü, X., \& Saka, S. (2012). New insights on monosaccharides' isomerization, dehydration and fragmentation in hot-compressed water. Journal of Supercritical Fluids, 61, 146-156.

Lu, Y., Levin, G. V., \& Donner, T. W. (2008). Tagatose, a new antidiabetic and obesity control drug. Diabetes, Obesity and
Metabolism, 10(2), 109-134.

Marugg, J. E., Tromp, M., Kuyl-Yeheshiely, E., van der Marel, G. A., \& van Boom, J. H. (1986). A convenient and general approach to the synthesis of properly protected D-nucleoside-3'-hydrogenphosphonates via phosphite intermediates. Tetrahedron Letters, 27(23), 2661-2664.

Mendicino, J. F. (1960). Effect of borate on the alkali-catalyzed isomerization of sugars. Journal of the American Chemical Society, 82, 4975-4979.

Moliner, M., Roman-Leshkov, Y., \& Davis, M. E. (2010). Tin-containing zeolites are highly active catalysts for the isomerization of glucose in water. Proceedings of the National Academy of Sciences of the United States of America, 107(14), 6164-6168.

Tipson, R. S., \& Brady Jr, R. F. (1969). Synthesis of the two D-2-pentuloses new derivatives of D-erythro-pentulose. Carbohydrate Research, 10(4), 549-563.

Usuki, C., Kimura, Y., \& Adachi, S. (2007). Isomerization of hexoses in subcritical water. Food Science and Technology Research, 13(3), 205-209.

Visuri, K., \& Klibanov, A. M. (1987). Enzymatic production of high fructose corn syrup (HFCS) containing 55\% fructose in aqueous ethanol. Biotechnology and Bioengineering, 30(7), 917-920.

Vourinen, T., \& Serianni, A. S. (1990). Synthesis of D-erythro-2-pentulose and D-threo-2-pentulose and analysis of the ${ }^{13} \mathrm{C}$ - and ${ }^{1} \mathrm{H}$-n.m.r. spectra of the $1-{ }^{13} \mathrm{C}$ - and $2-{ }^{13} \mathrm{C}$-substituted sugars. Carbohydrate Research, 209, 13-31.

Vourinen, T., \& Sjostrom, E. (1982). Kinetics of alkali-catalyzed isomerization of D-glucose and D-fructose in ethanol-water solutions. Carbohydrate Research, 108, 23-29.

de Wit, G., Kieboom, A. P. G., \& van Bekkum, H. (1979). Enolization and isomerization of monosaccharides in aqueous, alkaline solution. Carbohydrate Research, 74, 157-175.

Yamauchi, T., Fukushima, K., Yanagihara, R., Osanai, S., \& Yoshikawa S. (1990). Epimerization and isomerization of various monosaccharides using metal-diamine systems. Carbohydrate Research, 204, 233-239. 